\title{
Surface-enhanced Raman Scattering Detection of Nucleic Acids exhibiting Sterically Accessible Guanines using Ruthenium-polypyridyl Reagents
}

Miguel Martínez-Calvo, Luca Guerrini, Jéssica Rodríguez, Ramón A. ÁlvarezPuebla and José L. Mascareñas

\section{Peer reviewed version}

This is the peer reviewed version of the following article: Martínez-Calvo, M.; Guerrini, L.; Rodríguez, J.; Álvarez Puebla, R. A.; Mascareñas, J. L. (2020), Surface-enhanced Raman Scattering Detection of Nucleic Acids exhibiting Sterically Accessible Guanines using Ruthenium-polypyridyl Reagents. J. Phys. Chem. Lett., 11: 7218-7223, which has been published in final form at https://doi.org/10.1021/acs.jpclett.0c02148. This article may be used for non-commercial purposes in accordance with ACS Terms and Conditions for Use of Self-Archived Versions.

\section{How to cite:}

This is the peer reviewed version of the following article: Martínez-Calvo, M.; Guerrini, L.; Rodríguez, J.; Álvarez Puebla, R. A.; Mascareñas, J. L. (2020), Surface-enhanced Raman Scattering Detection of Nucleic Acids exhibiting Sterically Accessible Guanines using Ruthenium-polypyridyl Reagents. J. Phys. Chem. Lett., 11: 7218-7223. doi: 10.1021/acs.jpclett.0c02148

\section{Copyright information:}

(C) 2020 ACS. This article may be used for non-commercial purposes in accordance with ACS Terms and Conditions for self-archiving 


\title{
Surface-enhanced Raman Scattering Detection of
}

\section{Nucleic Acids exhibiting Sterically Accessible \\ Guanines using Ruthenium-polypyridyl Reagents}

\author{
Miguel Martínez-Calvo, ${ }^{a, c \dagger}$ Luca Guerrini, ${ }^{* b \dagger}$ Jéssica Rodríguez, ${ }^{a}$ Ramón A. Álvarez-Puebla ${ }^{* b, d}$ \\ and José L. Mascareñas *a
}

${ }^{a}$ Centro Singular de Investigación en Química Biolóxica e Materiais Moleculares (CIQUS) and Departamento de Química Orgánica. Universidade de Santiago de Compostela.Rúa Jenaro de la Fuente s/n, 15782 Santiago de Compostela, Spain.

${ }^{b}$ Universitat Rovira i Virgili, Departament de Química Física i Inorgànica, EmaS. Carrer de Marcel-lí Domingo s/n, 43007 Tarragona, Spain. E-mail: luca.guerrini@urv.cat; ramon.alvarez@urv.cat

${ }^{c}$ Centro de Investigaciones Avanzadas (CICA), AE CICA-INIBIC, Departamento de Química, Facultade de Ciencias. Universidade da Coruña. Rúa As Carballeiras s/n, 15071 A Coruña, Galicia, Spain.

${ }^{d}$ ICREA, Passeig Lluís Companys 23, 08010 Barcelona, Spain

$†$ These authors contributed equally 


\section{AUTHOR INFORMATION}

\section{Corresponding Authors}

Luca Guerrini, e-mail: luca.guerrini@urv.cat

Ramon A. Alvarez-Puebla, e-mail: ramon.alvarez@urv.cat

Jose Luis Mascareñas, e-mail: joseluis.mascarenas@usc.es

ACS Paragon Plus Environment 


\begin{abstract}
Here, we report the application of surface-enhanced Raman scattering (SERS) spectroscopy as a rapid and practical tool for assessing the formation of coordinative adducts between nucleic acid guanines and ruthenium polypyridyl reagents. The technology provides a practical approach for the wash-free and quick identification of nucleic acid structures exhibiting sterically accessible guanines. This is demonstrated for the detection of a quadruplex-forming sequence present in the promoter region of the $c-m y c$ oncogene, which exhibits a non-paired, reactive guanine at a flanking position of the G-quartets.
\end{abstract}

\title{
TOC GRAPHICS
}

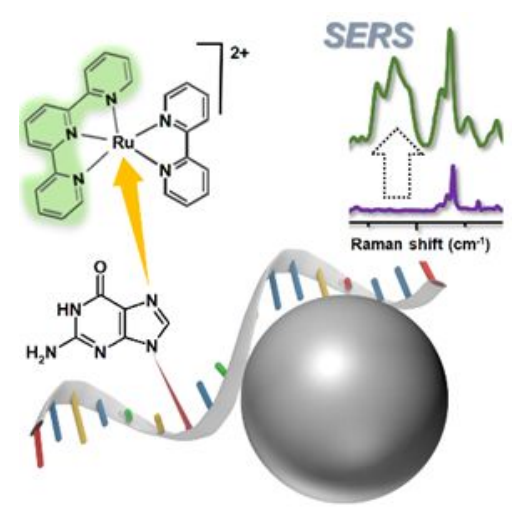

KEYWORDS: ruthenium • metalation • surface-enhanced Raman $\bullet$ DNA • plasmonic 
DNA-metalating agents (e.g., cis-platinum and derivatives) are widely used as chemotherapeutic drugs for the treatment of cancers. ${ }^{1}$ However, the toxicity and resistance problems associated with these therapies has prompted the development of alternatives based on other metals, which might exhibit enhanced selectivity profiles. ${ }^{2-3}$ Ruthenium is especially appealing owing to a wide repertoire of oxidation states and coordination geometries, together with the possibility of fine-tuning the reactivity by a proper ligand selection, and/or by irradiation with light. ${ }^{4}$ Therefore, several metalating agents based on ruthenium have been developed, some of which exhibit attractive antitumoral profiles..$^{3,5}$ Most of these bioactive derivatives bind double-stranded DNA (dsDNA) by coordination to the nitrogen at position 7 (N7) of guanines, which is accessible through the DNA major groove. ${ }^{6-7}$ Unfortunately, these ruthenium derivatives tend to present a promiscuous reactivity.

Recently, we have shown that a cationic octahedral $\mathrm{Ru}(\mathrm{II})$ complex featuring terpyridine and bipyridine ligands, $[\mathrm{Ru}(t p y)(b p y) \mathrm{Cl}]^{+}$, can react with the $\mathrm{N} 7$ of guanosine monophosphate (GMP). ${ }^{4,8}$ The reaction is mediated by the formation of an aquo reaction intermediate $\left[\mathrm{Ru}(t p y)(b p y) \mathrm{OH}_{2}\right]^{+2}\left(\mathbf{R u}_{\mathbf{a q}}\right)$, and accelerated by irradiation with light (at $\left.c a .430 \mathrm{~nm}\right) .{ }^{9}$ Remarkably, dsDNAs remained unreactive, likely because of the bulkiness of the reagent; however, this ruthenium complex smoothly reacts with solvent-exposed guanines present in flanking positions of specific G-quadruplexes (GQ), like one in the promoter region of the oncogene $c-M y c .^{8}$

$c-M y c$ is known to play a crucial role in the development of many cancers, ${ }^{10}$ and therefore it has been recognized as a highly valuable target for chemotherapy, as well as a cancer biomarker. ${ }^{11}$ In this context, $\mathbf{R} \mathbf{u}_{\mathbf{a q}}$ promises to be a good lead for the development of $M y c$ targeting anticancer agents. Beyond that, the selective reactivity of the metal complex with the $c$ - 
Myc GQ might allow the detection of this important DNA sequence, provided a reliable and sensible analytic method to monitor the metalation process could be implemented.

In this context, we envisioned that the pyridine ligands of the ruthenium complex may represent suitable probes to monitor the interaction process by surface-enhanced Raman spectroscopy (SERS). SERS is a powerful analytical technique that has demonstrated utility for the detection and structural characterization of many biomolecules, including nucleic acids. ${ }^{12-17}$ However, it has not been used for investigating nucleic acid ruthenations, except for one isolated example restricted to DNA duplexes. ${ }^{18}$

Here, we demonstrate that the interaction of the ruthenium complex $\mathbf{R} \mathbf{u}_{\mathbf{a q}}$ with the Gquadruplex motive present in the promoter of $c-M y c$ can be readily identified by SERS, using positively-charged silver colloids as plasmonic substrates. Most notably, the terpyridine moiety (tpy) at the ruthenium provides an excellent vibrational fingerprint to discriminate binding events as a result of its structural deformation upon metalation of the nucleic acids. The approach can be extended to guanine rich single-stranded DNAs, as well as RNAs, and even allows to assess whether the RNA sequences feature 8-oxoguanines instead of guanines. Overall, the technology provides a rapid, wash-free way to screen nucleic acid structures which feature reactive, sterically accessible guanines (Scheme 1), paving the way for its potential application in early diagnosis and drug discovery. 


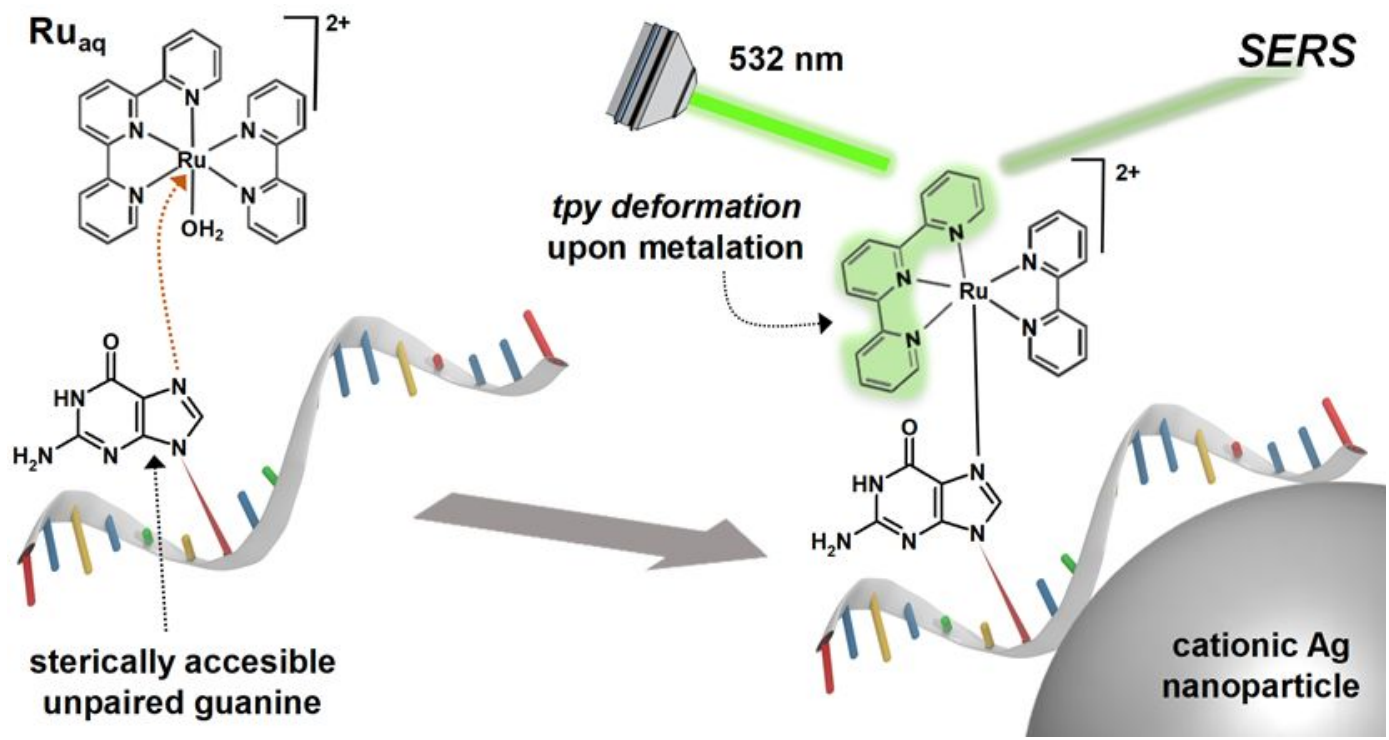

Scheme 1. Outline of the SERS monitoring process.

The synthesis of cationic silver colloids (AgSp) was achieved upon reduction of $\mathrm{Ag}^{+}$ions in the presence of spermine tetrahydrochloride, which yields a suspension of nanoparticles of ca. 23 $\mathrm{nm}$ diameter with a localized surface plasmon resonance (LSPR) centered at ca. $391 \mathrm{~nm}$ (Figure 1 and Figure S1A). The linear cationic spermine molecules are retained at the silver surface via interaction with metallic-bound $\mathrm{Cl}^{-}$anions, yielding an outer spermine ad-layer that confers an overall positive charge to the nanoparticles $(\zeta$ potential of ca. $+40 \mathrm{mV}) .{ }^{19}$ The efficient electrostatic interaction between the surface spermine molecules and the negatively charged backbone of DNA enables a rapid aggregation of the nanoparticles upon addition of minute amounts of nucleic acids, as revealed by the red-shift and broadening of the LSPR (Figure 1). This aggregation results in stable clusters in suspension which yield intense and reproducible SERS spectra. ${ }^{19-20}$ Helpfully, the SERS background spectrum of the colloids does not show any significant features in the spectral range of interest for the DNA analysis (Figure S1B). 


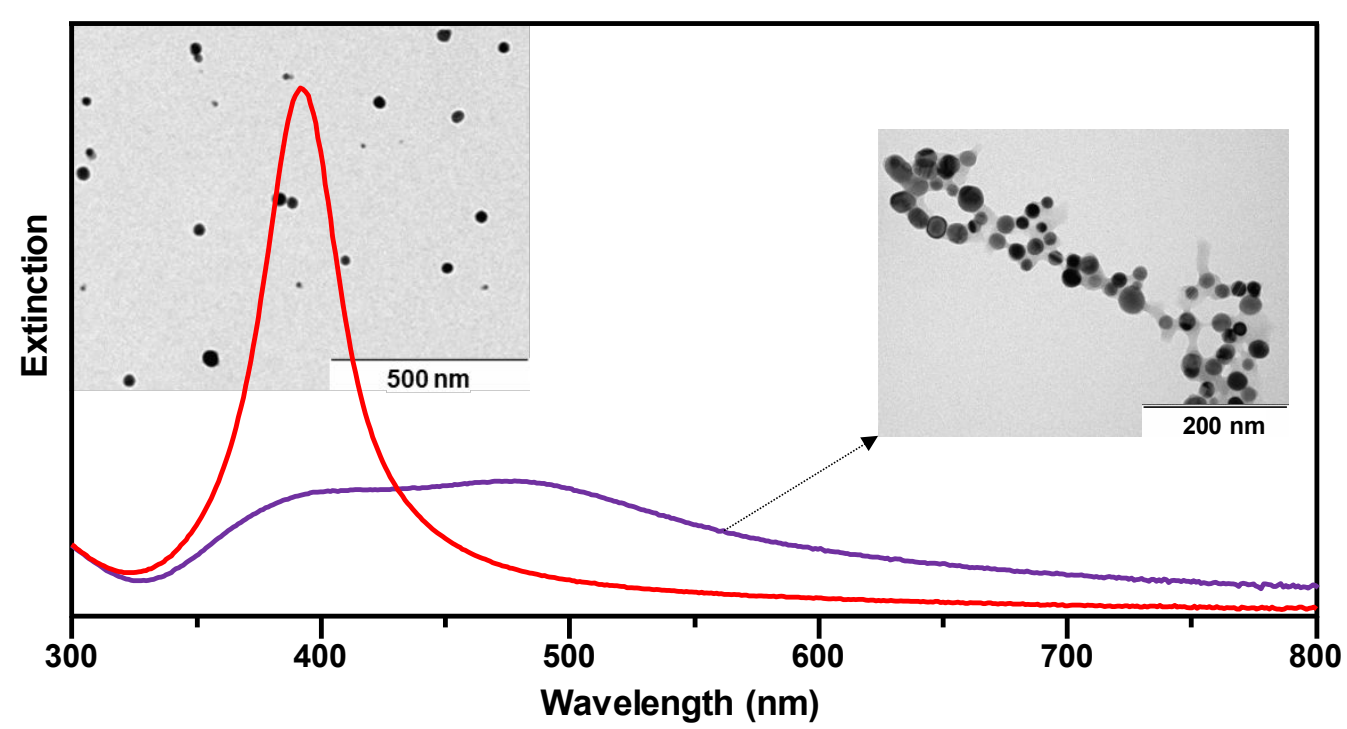

Figure 1. Extinction spectrum of positively charged silver colloids before (red curve) and after (purple curve) the addition of MYC (final DNA concentration in the sample ca. $0.6 \mu \mathrm{M}$ ).

Representative TEM images are also included (samples were diluted before the deposition onto the copper grid to mitigate the problem of unspecific particle agglomeration). Additional TEM characterization of the undiluted AgSp colloids is reported in Figure S1A.

As a representative of the $c-M y c$ promoter site, we used the sequence $M Y C$ (Figure 2), which folds in solution into the required GQ quadruplex, as confirmed by circular dichroism (Figure S2A). The SERS spectrum presents the expected signals for a nucleic acid structure (blue line, Figure 2). Gratifyingly, mixing of $\boldsymbol{M Y C}$ with $\mathbf{R} \mathbf{u}_{\mathbf{a q}}$ in phosphate-buffered saline (PBS) overnight at room temperature (DNA/R $\mathbf{u}_{\mathbf{a q}}$ molar ratio 1:3) gave rise to a new spectrum dominated by features of the ruthenium complex $\left(\boldsymbol{M Y C} / \mathbf{R} \mathbf{u}_{\mathbf{a q}}\right.$, green line), and different to that resulting from $\mathbf{R} \mathbf{u}_{\mathbf{a q}}$ alone (red line). As previously reported, ruthenium cationic complexes typically adsorb on silver nanoparticles, likely via electrostatic interactions with superficial chloride anions, leading 
to minimal perturbation of their ground state molecular structure. ${ }^{21}$ It is worth noting that the reactivity of $\mathbf{R} \mathbf{u}_{\mathrm{aq}}$ with $\boldsymbol{M Y C}$ was confirmed by HPLC analysis (Figure S2B). ${ }^{8}$

The signals of the metallocomplex in $\boldsymbol{M Y C} / \mathbf{R} \mathbf{u}_{\mathrm{aq}}$ are so strong that the DNA contributions are even difficult to observe (visible DNA features are marked by an asterisk in Figure 2). This can be explained as follows. The SERS experiments are carried out using an excitation wavelength of $532 \mathrm{~nm}$ excitation, which coincides with the red tail of the metal-to-ligand charge transfer transition $(\mathrm{MLCT}) R u(d) \rightarrow \operatorname{tpy}\left(\pi^{*}\right)$ (Figure $\left.\mathrm{S} 1 \mathrm{C}\right) .{ }^{22}$ The selective resonance with this MLCT transition provides an additional enhancement to tpy features (i.e., surface-enhanced resonance Raman, SERRS) which fully dominate the spectral profile, ${ }^{22}$ with intensities well above those of the DNA bands. This can even be better appreciated by removing the residual sequencedependent DNA contributions from the spectra, using a digital subtraction of the SERS signal of $M Y C$ from the corresponding mixture with $\mathbf{R} \mathbf{u}_{\mathrm{aq}}\left(\boldsymbol{M Y C} / \mathbf{R} \mathbf{u}_{\mathrm{aq}}-\boldsymbol{M Y C}\right.$, Figure 2, bottom). The difference spectrum displays major alterations with respect to that of $\mathbf{R} \mathbf{u}_{\mathbf{a q}}$ alone, most notably a dramatic increase of the tpy associated features at $518 \mathrm{~cm}^{-1}$ (out-of-plane ring torsion), $555 \mathrm{~cm}^{-1}$ (in-plane bridge bending) and $766 \mathrm{~cm}^{-1}$ (out-of-plane $\mathrm{CH}$ bending/ring torsion) as compared to the bands at higher wavelengths, such as the in-plane ring stretching at $1487 \mathrm{~cm}^{-1} .^{23} \mathrm{We}$ also observe the emergence of two bands at 648 and $699 \mathrm{~cm}^{-1}$, which can be tentatively ascribed to inplane ring bending and resulting from linear combinations of inner/outer tpy ring deformations. ${ }^{23}$ This overall trend of spectral changes has been previously observed in isolated ruthenium terpyridyl complexes as a result of ligand substitution. ${ }^{22}$ As the spectral reshaping is mostly associated with Raman bands that are sensitive to the geometry of the MLCT resonance state, we can infer that upon guanine coordination there is a distortion in the tpy structure. Other features, 
such as the band at $1487 \mathrm{~cm}^{-1}$, do not provide structural information likely because they are enhanced through different mechanisms. ${ }^{23}$

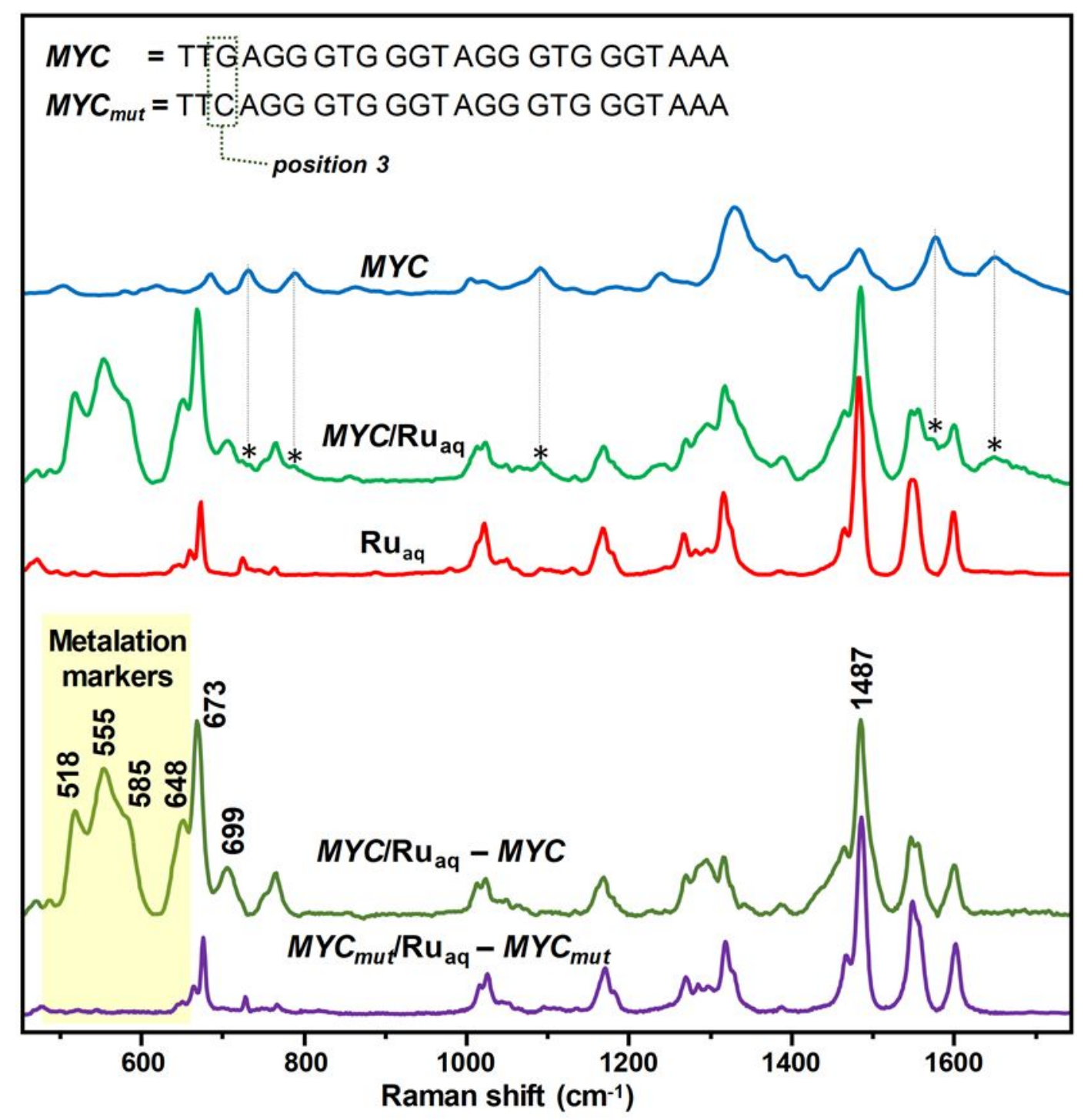

Figure 2. SERS spectra (baseline corrected) of the different samples (upper), and after subtraction of those resulting from the oligos (bottom). The SERS spectrum of $\mathrm{Ru}_{\mathrm{aq}}$ was obtained on $\mathrm{AgSp}$ upon addition of an aliquot of a $0.5 \mathrm{M} \mathrm{MgSO}_{4}$ solution acting as a passive aggregating agent (i.e., leads to colloidal aggregation without altering the chemical surface properties of the nanoparticles). $\mathrm{DNA} / \mathrm{Ru}_{\mathrm{aq}}$ molar ratio $=1: 3$; DNA concentration in the sample ca. $0.6 \mu \mathrm{M}$. All 
spectra were normalized to the $1487 \mathrm{~cm}^{-1}$ band. Marker bands for ruthenation are highlighted in yellow.

We carried out identical experiments with a $c$-myc mutated derivative $\left(\boldsymbol{M Y} \boldsymbol{C}_{\boldsymbol{m} u t}\right)$, in which the reactive guanine residue in position 3 (G3) was replaced by cytosine (Figure 2). The resulting difference SERS spectrum $\left(\boldsymbol{M Y C _ { m u t }} / \mathbf{R} \mathbf{u}_{\mathrm{aq}}-\boldsymbol{M Y C _ { m u t }}\right)$ presents a vibrational profile that almost entirely matches that of $\mathbf{R} \mathbf{u}_{\mathbf{a q}}$ alone, suggesting that no apparent interaction between the oligonucleotide and the Ru complex has occurred. As expected, $\boldsymbol{M Y} \boldsymbol{C}_{\boldsymbol{m} u t}$ also presents a quadruplex structure in solution (Figure S2A).

Not surprisingly, mixing guanine-rich double-stranded DNAs ( $d s$ DNA) with $\mathbf{R u}_{\mathbf{a q}}$, under the same experimental conditions, did not promote any change in the SERS spectra (Figure S3). We also tested duplexes containing internal $\left(d s \mathrm{DNA}_{\mathrm{m} 1}\right)$ and external $\left(d s \mathrm{DNA}_{\mathrm{m} 2}\right) \mathrm{G} \cdot \mathrm{A}$ mismatches, and again we did not observe the tpy SERS signals resulting from metalation (Figure S3). These results are consistent with the inability of the bulky ruthenium complex to approach and appropriately align with the nucleophilic nitrogen of the sterically constrained guanines within the DNA double helix.

In contrast, single-stranded oligonucleotides ( $s S$ DNA) featuring guanine bases do yield SERS bands similar to those observed for $M Y C$ (Figure 3 and S4). Specifically, the selective binding of $\mathbf{R} \mathbf{u}_{\mathbf{a q}}$ to unpaired guanines in oligonucleotides was verified for 18-mer ssDNAs exhibiting several $\mathrm{G}$ bases in their structures $\left(\boldsymbol{s s} \boldsymbol{G}_{3} \boldsymbol{T}_{3}\right.$ and $\left.\boldsymbol{s s} \boldsymbol{G}_{3} \boldsymbol{A}_{3}\right)$. These sequences were selected using computational tools that predict their inability to form G-quadruplex structures. ${ }^{24}$ The SERS spectra of $\mathrm{ssG}_{3} \mathrm{~T}_{3}$ and $\mathrm{ssG}_{3} \mathrm{~A}_{3}$ in the presence of $\mathrm{Ru}_{\mathrm{aq}}$ present vibrational profiles that largely overlap with that of $\boldsymbol{M Y C} / \mathbf{R} \mathbf{u}_{\mathrm{aq}}$. However, with guanine-free $s s \mathrm{DNAs}\left(\boldsymbol{s s} \boldsymbol{C}_{3} \boldsymbol{T}_{3}\right.$. and $\left.\boldsymbol{s s} \boldsymbol{C}_{3} \boldsymbol{A}_{3}\right)$, there 
are no changes, confirming the chemoselectivity of $\mathbf{R} \mathbf{u}_{\mathrm{aq}}$ towards guanines (sterically accessible).

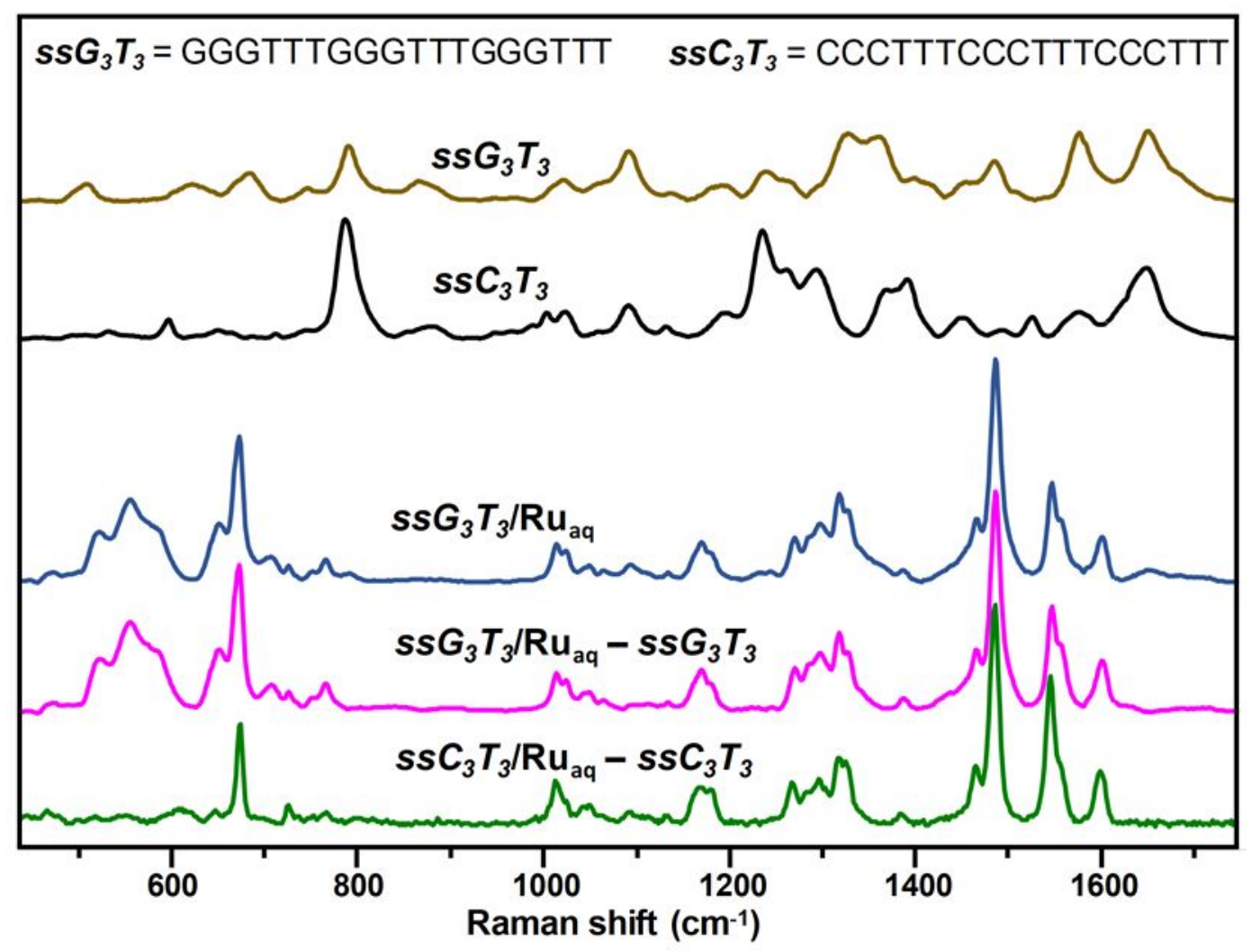

Figure 3. SERS spectra of single-stranded DNAs and the corresponding mixtures after reaction with $\mathbf{R} \mathbf{u}_{\mathbf{a q}}\left(\mathrm{DNA} / \mathbf{R} \mathbf{u}_{\mathbf{a q}}\right.$ molar ratio $=1: 3$, DNA concentration in the sample ca. $\left.0.6 \mu \mathrm{M}\right)$. Difference SERS spectra of $\mathbf{s s G}_{\mathbf{3}} \mathbf{A}_{\mathbf{3}}$ and $\mathbf{s s} \mathbf{G}_{\mathbf{3}} \mathbf{T}_{\mathbf{3}}$ mixtures with $\mathbf{R} \mathbf{u}_{\mathbf{a q}}$ are also illustrated. All spectra were normalized to the $1487 \mathrm{~cm}^{-1}$ band.

Interestingly, we obtained qualitatively analogous results by using a different incubation protocol, i.e. first combining the oligonucleotides with the colloids, and then adding the complex $\mathbf{R} \mathbf{u}_{\mathbf{a q}}$ to the suspension (Figure S5). On the contrary, when the ruthenium complex is incubated beforehand with AgSp, the subsequent addition of DNA does not lead to alterations in the tpy 
marker bands, suggesting that the adhesion of $\mathbf{R} \mathbf{u}_{\mathbf{a q}}$ onto the metallic surface largely suppress its metalating reactivity.

We also performed SERS measurements with $\boldsymbol{s} \boldsymbol{s} \boldsymbol{G}_{3} \boldsymbol{T}_{3}$ strands in the presence of a ruthenium derivative in which the reactive position is blocked by coordination to the sulfur atom of a methionine $[\operatorname{Ru}(t p y)(b p y) m e t]^{+2}\left(\mathbf{R} \mathbf{u}_{\text {met }}\right.$, met $=$ methionine $) .{ }^{8}$ In consonance with the inertness of this complex, we observed negligible spectral alterations even after extended illumination times (Figure S6).

All these findings confirm that the vibrational profile of the terpyridine (tpy) ligand undergoes a substantial change upon coordination of the ruthenium complex to the N7 of exposed guanines in different types of oligonucleotides. It is also worth noting that the very large Raman crosssection of the tpy ligand under $532 \mathrm{~nm}$ excitation leads to resonantly-enhanced contributions which largely overlap those of guanine residues, making, in turn, undistinguishable the expected spectral changes of $\mathrm{G}$ bands sensitive to N-7 alterations. ${ }^{25}$

While all the above results involve DNA, we also questioned whether the approach could be extended to guanine-rich RNAs. RNA presents a wider range of conformations, mostly nonduplexes, indicating that its guanines might be available for coordination with the bulky ruthenium reagent. ${ }^{26}$ Indeed, using the standard analysis conditions, we observed that mixing $\mathbf{R u}_{\mathbf{a q}}$ with the 21-mer RNA $\boldsymbol{m i R N} \boldsymbol{A}$ generates a SERS spectrum exhibiting the expected features associated with a metalation reaction (Figure 4). Interestingly, the spectral contour of the SERS marker bands of the tpy moiety appears different from that observed in the case of DNA, suggesting that the structural distortion of tpy takes place differently. This can be tentatively 
ascribed to the different environment provided by the RNA, in part because of a rather distinct backbone conformation than DNA. ${ }^{27}$

As SERS sensing depends on the presence of the nucleophilic nitrogen at the position 7 of guanine, we reasoned that base analogues lacking this feature should not be able to react with the ruthenium reagent. This should be the case for 8-oxoguanines (Figure 3), which are biologically significant derivatives that result from the oxidative damage of guanines. ${ }^{28-29}$ This is becoming particularly intriguing for the case of RNA, in which the oxidation of guanine to 8-oxoguanine has been correlated with the progression of neurodegenerative diseases and cancer. ${ }^{30-32}$ Gratifyingly, performing the standard SERS analysis with $\boldsymbol{m i R N} \boldsymbol{A}_{\boldsymbol{O}}$, which is similar to $\boldsymbol{m i R} \boldsymbol{N} \boldsymbol{A}$, but contains oxoguanines instead of native guanines, we observed the absence of signals below $600 \mathrm{~cm}^{-1}$.

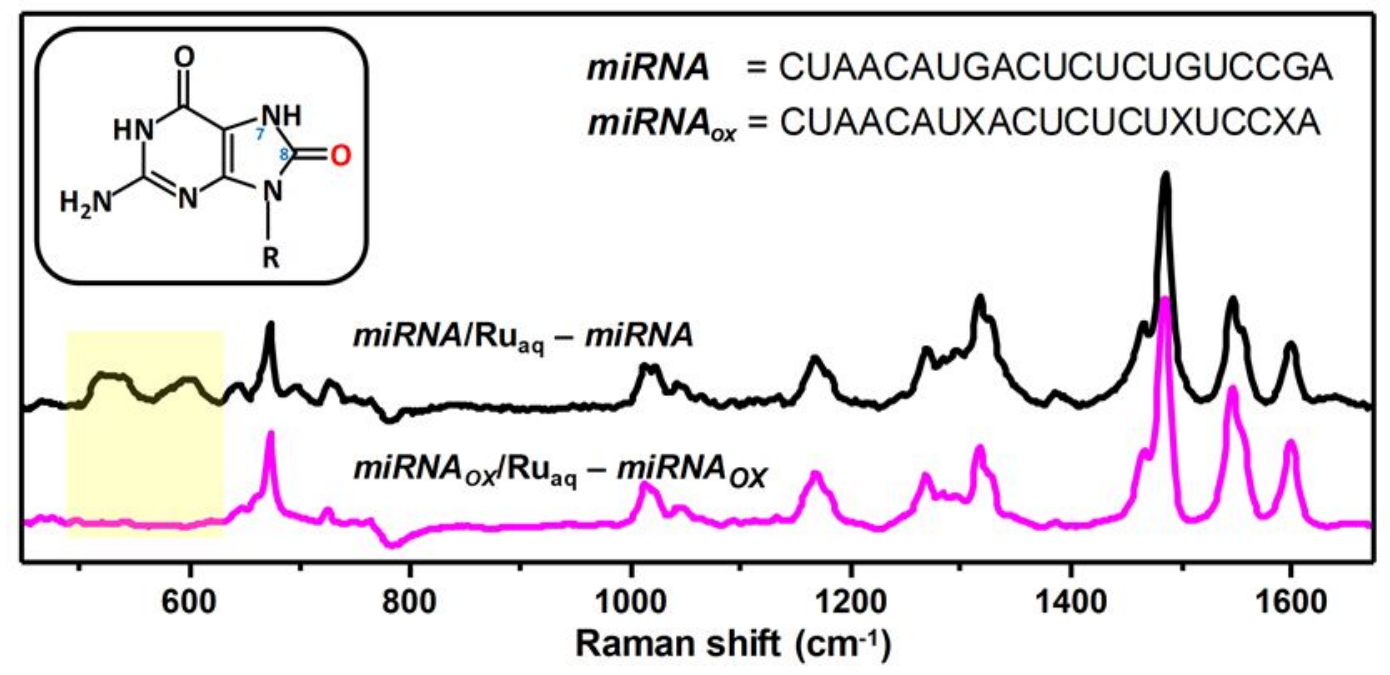

Figure 4. Difference SERS spectra (baseline corrected) of the miRNA/Ru $u_{a q}$ and $m i R N A_{O X} / R_{a q}$ samples $(\mathrm{RNA} / \mathrm{Ru}$ molar ratio $=1: 3, \mathrm{RNA}$ concentration in the sample ca. $0.6 \mu \mathrm{M})$. Inset: molecular structure of the 8-oxoguanine nucleobase (X). 
All SERS measurements illustrated in the study were performed adopting a static configuration, ${ }^{19}$ and relatively large volumes of colloids $(130 \mu \mathrm{L})$ with a final DNA concentration of $c a .0 .6 \mu \mathrm{M}$. This set-up offers a straightforward and very simple approach for acquiring highly reproducible SERS spectra. As previously illustrated, ${ }^{19,} 33$ reduction of the DNA concentration is not a practical approach for decreasing the limit of detection, as it negatively affects the generation of interparticle hot spots (DNA acts itself as the aggregating agent). Conversely, integration of this detection protocol with microfluidics offers the possibility of dramatically reducing the required amount of sample for the SERS analysis (less than 300 picograms of DNA) while enabling automation of the whole procedure. ${ }^{34}$ Nonetheless, it is worth stressing that current methods for detection of metalated DNA, such as HPLC and CD, require much larger amounts of nucleic acids, with sample typically in the 10-100 $\mu \mathrm{M}$ range of DNA concentration. ${ }^{8,35}$

In summary, we have implemented a SERS technology for the wash-free detection of nucleic acid structures containing sterically accessible guanines. The method lies on the ability of the bulky ruthenium polypyridine compound $\left[\mathrm{Ru}(t p y)(b p y) \mathrm{OH}_{2}\right]^{+2}$ to coordinate the nucleophilic $\mathrm{N} 7$ of exposed guanines. Specifically, the tpy ligand represents an excellent SERS probe, because after coordination of the metal to the nucleic acids it undergoes a SERS-signaling distortion that can be easily detected. Importantly, the method can be used for sensing relevant secondary structures, such as the G-quadruplex forming DNA sequence present in the promoter of the oncogene $c-m y c$, a biomarker of cancer. Altogether, the approach promises to nurture new advances in the study and development of selective DNA metalating agents and practical diagnosis tools as well as the screening of anticancer drugs.

\section{ASSOCIATED CONTENT}


Supporting Information. Experimental procedures, circular dichroism and additional SERS data.

\section{AUTHOR INFORMATION}

\section{Notes}

The authors declare no competing financial interests.

\section{ACKNOWLEDGMENT}

We are thankful for the financial support from the Xunta de Galicia (Centro singular de investigación de Galicia accreditation 2019-2022, ED431G 2019/03) and the European Union (European Regional Development Fund - ERDF). We also thank the support given by the Spanish grant SAF2013-41943-R and SAF2016-76689-R, the Xunta de Galicia (grants 2015CP082, ED431C 2017/19,), the Spanish Ministry de Economia y Competitividad (CTQ201788648R and RYC-2016-20331), the Generalitat de Cataluña (2017SGR883), the Universitat Rovira i Virgili (2019PFR-URV-B2-02), the Universitat Rovira i Virgili and Banco Santander (2017EXIT-08) and the European Research Council (Advanced Grant No. 340055). MMC thanks the Ministerio de Economía y Competitividad for the Postdoctoral fellowship (IJCI-201419326) and the Ministerio de Ciencia e Innovación and Ministerio de Universidades for the Distinguished Researcher contract "Beatriz Galindo" (BEAGAL18/00144). JR thanks to Xunta de Galicia for her predoctoral fellowship.

\section{REFERENCES}

(1) Kelland, L. The Resurgence of Platinum-Based Cancer Chemotherapy. Nat. Rev. Cancer 2007, 7, 573. 
(2) Wheate, N. J.; Walker, S.; Craig, G. E.; Oun, R. The Status of Platinum Anticancer Drugs in the Clinic and in Clinical Trials. Dalton Trans. 2010, 39, 8113-8127.

(3) Muhammad, N.; Guo, Z. J. Metal-Based Anticancer Chemotherapeutic Agents. Curr. Opin. Chem. Biol. 2014, 19, 144-153.

(4) Brabec, V.; Kasparkova, J. Ruthenium Coordination Compounds of Biological and Biomedical Significance. DNA Binding Agents. Coord. Chem. Rev. 2018, 376, 75-94.

(5) Zeng, L. L.; Gupta, P.; Chen, Y. L.; Wang, E. J.; Ji, L. N.; Chao, H.; Chen, Z. S. The Development of Anticancer Ruthenium(II) Complexes: From Single Molecule Compounds to Nanomaterials. Chem. Soc. Rev. 2017, 46, 5771-5804.

(6) Betanzos-Lara, S.; Salassa, L.; Habtemariam, A.; Novakova, O.; Pizarro, A. M.; Clarkson, G. J.; Liskova, B.; Brabec, V.; Sadler, P. J. Photoactivatable Organometallic Pyridyl Ruthenium(II) Arene Complexes. Organometallics 2012, 31, 3466-3479.

(7) Peacock, A. F. A.; Sadler, P. J. Medicinal Organometallic Chemistry: Designing Metal Arene Complexes as Anticancer Agents. Chem. Asian J. 2008, 3, 1890-1899.

(8) Rodríguez, J.; Mosquera, J.; Couceiro, J. R.; Vázquez, M. E.; Mascareñas, J. L. Ruthenation of Non-Stacked Guanines in DNA G-Quadruplex Structures: Enhancement of CMyc Expression. Angew. Chem.-Int. Edit. 2016, 55, 15615-15618.

(9) Goldbach, R. E.; Rodriguez-Garcia, I.; van Lenthe, J. H.; Siegler, M. A.; Bonnet, S. NAcetylmethionine and Biotin as Photocleavable Protective Groups for Ruthenium Polypyridyl Complexes. Chem. Eur. J. 2011, 17, 9924-9929.

(10) Dang, C. V.; Resar, L. M. S.; Emison, E.; Kim, S.; Li, Q.; Prescott, J. E.; Wonsey, D.; Zeller, K. Function of the c-Myc Oncogenic Transcription Factor. Exp. Cell Res. 1999, 253, 63 77.

(11) Chen, B. J.; Wu, Y. L.; Tanaka, Y.; Zhang, W. Small Molecules Targeting c-Myc Oncogene: Promising Anti-Cancer Therapeutics. Int. J. Biol. Sci. 2014, 10, 1084-1096. (12) Schlücker, S. Surface-enhanced Raman Spectroscopy: Concepts and Chemical Applications. Angew. Chem.-Int. Edit. 2014, 53, 4756-4795.

(13) Garcia-Rico, E.; Alvarez-Puebla, R. A.; Guerrini, L. Direct Surface-enhanced Raman Scattering (SERS) Spectroscopy of Nucleic Acids: From Fundamental Studies to Real-Life Applications. Chem. Soc. Rev. 2018, 47, 4909-4923. 
(14) Zong, C.; Xu, M. X.; Xu, L. J.; Wei, T.; Ma, X.; Zheng, X. S.; Hu, R.; Ren, B. SurfaceEnhanced Raman Spectroscopy for Bioanalysis: Reliability and Challenges. Chem. Rev. 2018, $118,4946-4980$.

(15) Cialla-May, D.; Zheng, X. S.; Weber, K.; Popp, J. Recent Progress in Surface-enhanced Raman Spectroscopy for Biological and Biomedical Applications: From Cells to Clinics. Chem. Soc. Rev. 2017, 46, 3945-3961.

(16) Pazos, E.; Garcia-Algar, M.; Penas, C.; Nazarenus, M.; Torruella, A.; Pazos-Perez, N.; Guerrini, L.; Vázquez, M. E.; Garcia-Rico, E.; Mascareñas, J. L.; Alvarez-Puebla, R. A. Surfaceenhanced Raman Scattering Surface Selection Rules for the Proteomic Liquid Biopsy in Real Samples: Efficient Detection of the Oncoprotein C-Myc. J. Am. Chem. Soc. 2016, 138, 1420614209.

(17) Li, Y.; Han, X.; Zhou, S.; Yan, Y.; Xiang, X.; Zhao, B.; Guo, X. Structural Features of DNA G-Quadruplexes Revealed by Surface-enhanced Raman Spectroscopy. J. Phys. Chem. Lett. 2018.

(18) Bugarcic, T.; Nováková, O.; Halámiková, A.; Zerzánková, L.; Vrána, O.; Kašpárková, J.; Habtemariam, A.; Parsons, S.; Sadler, P. J.; Brabec, V. Cytotoxicity, Cellular Uptake, and DNA Interactions of New Monodentate Ruthenium(II) Complexes Containing Terphenyl Arenes. $J$. Med. Chem. 2008, 51, 5310-5319.

(19) Guerrini, L.; Krpetić, Ž.; van Lierop, D.; Alvarez-Puebla, R. A.; Graham, D. Direct Surface-enhanced Raman Scattering Analysis of DNA Duplexes. Angew. Chem.-Int. Edit. 2015, 54, 1144-1148.

(20) Morla-Folch, J.; Alvarez-Puebla, R. A.; Guerrini, L. Direct Quantification of DNA Base Composition by Surface-enhanced Raman Scattering Spectroscopy. J. Phys. Chem. Lett. 2016, 7, 3037-3041.

(21) Šloufová, I.; Vlčková, B.; Procházka, M.; Svoboda, J.; Vohlídal, J. Comparison of SERRS and RRS Excitation Profiles of [Fe(Tpy)2]2+ (Tpy=2,2':6',2"-Terpyridine) Supported by DFT Calculations: Effect of the Electrostatic Bonding to Chloride-Modified Ag Nanoparticles on its Vibrational and Electronic Structure. J. Raman Spectrosc. 2014, 45, 338-348.

(22) Schneider, S.; Brehm, G.; Prenzel, C. J.; Jager, W.; Silva, M. I.; Burrows, H. D.; Formosinho, S. T. Vibrational Spectra, Normal Coordinate Analysis and Excited-State Lifetimes for a Series of Polypyridylruthenium(II) Complexes. J. Raman Spectrosc. 1996, 27, 163-175. 
(23) Hansen, P. W.; Jensen, P. W. Vibrational Studies on Bis-Terpyridine-Ruthenium(II) Complexes. Spectroc. Acta Pt. A-Molec. Biomolec. Spectr. 1994, 50, 169-183.

(24) Kikin, O.; D'Antonio, L.; Bagga, P. S. Qgrs Mapper: A Web-Based Server for Predicting G-Quadruplexes in Nucleotide Sequences. Nucleic Acids Res. 2006, 34, W676-W682.

(25) Masetti, M.; Xie, H.-n.; Krpetić, Ž.; Recanatini, M.; Alvarez-Puebla, R. A.; Guerrini, L. Revealing DNA Interactions with Exogenous Agents by Surface-enhanced Raman Scattering. $J$. Am. Chem. Soc. 2015, 137, 469-476.

(26) Li, X.; Gorle, A. K.; Ainsworth, T. D.; Heimann, K.; Woodward, C. E.; Grant Collins, J.; Richard Keene, F. RNA and DNA Binding of Inert Oligonuclear Ruthenium(II) Complexes in Live Eukaryotic Cells. Dalton Trans. 2015, 44, 3594-3603.

(27) Morla-Folch, J.; Xie, H.-n.; Alvarez-Puebla, R. A.; Guerrini, L. Fast Optical Chemical and Structural Classification of RNA. ACS Nano 2016, 10, 2834-2842.

(28) Dai, D. P.; Gan, W.; Hayakawa, H.; Zhu, J. L.; Zhang, X. Q.; Hu, G. X.; Xu, T.; Jiang, Z. L.; Zhang, Q.; Hu, X. D.; Nie, B.; Zhou, Y.; Li, J.; Zhou, X. Y.; Li, J.; Zhang, T. M.; He, Q.; Liu, D. G.; Chen, H. B.; Yang, N.; Zuo, P. P.; Zhang, Z. X.; Yang, H. M.; Wang, Y.; Wilson, S. H.; Zeng, Y. X.; Wang, J. Y.; Sekiguchi, M.; Cai, J. P. Transcriptional Mutagenesis Mediated by 8oxoG Induces Translational Errors in Mammalian Cells. Proc. Natl. Acad. Sci. U. S. A. 2018, $115,4218-4222$.

(29) Singh, S. K.; Szulik, M. W.; Ganguly, M.; Khutsishvili, I.; Stone, M. P.; Marky, L. A.; Gold, B. Characterization of DNA with an 8-Oxoguanine Modification. Nucleic Acids Res. 2011, 39, 6789-6801.

(30) Liu, Z.; Chen, X.; Li, Z.; Ye, W.; Ding, H.; Li, P.; Aung, L. H. H. Role of RNA Oxidation in Neurodegenerative Diseases. Int. J. Mol. Sci. 2020, 21, 1-14.

(31) Alenko, A.; Fleming, A. M.; Burrows, C. J. Reverse Transcription Past Products of Guanine Oxidation in Rna Leads to Insertion of A and C Opposite 8-Oxo-7,8-Dihydroguanine and $\mathrm{A}$ and $\mathrm{G}$ Opposite 5-Guanidinohydantoin and Spiroiminodihydantoin Diastereomers. Biochemistry 2017, 56, 5053-5064.

(32) Yan, L. L.; Simms, C. L.; McLoughlin, F.; Vierstra, R. D.; Zaher, H. S. Oxidation and Alkylation Stresses Activate Ribosome-Quality Control. Nat. Commun. 2019, 10.

(33) Gisbert-Quilis, P.; Masetti, M.; Morla-Folch, J.; Fitzgerald, J. M.; Pazos-Perez, N.; Garcia-Rico, E.; Giannini, V.; Alvarez-Puebla, R. A.; Guerrini, L. The Structure of Short and 
Genomic DNA at the Interparticle Junctions of Cationic Nanoparticles. Adv. Mater. Interfaces 2017, 4, 1700724.

(34) Morla-Folch, J.; Xie, H.-n.; Gisbert-Quilis, P.; Gómez-de Pedro, S.; Pazos-Perez, N.; Alvarez-Puebla, R. A.; Guerrini, L. Ultrasensitive Direct Quantification of Nucleobase Modifications in DNA by Surface-enhanced Raman Scattering: The Case of Cytosine. Angew. Chem.-Int. Edit. 2015, 54, 13650-13654.

(35) Liu, H.-K.; Wang, F.; Parkinson, J. A.; Bella, J.; Sadler, P. J. Ruthenation of Duplex and Single-Stranded D(CGGCCG) by Organometallic Anticancer Complexes. Chem. Eur. J. 2006, $12,6151-6165$. 


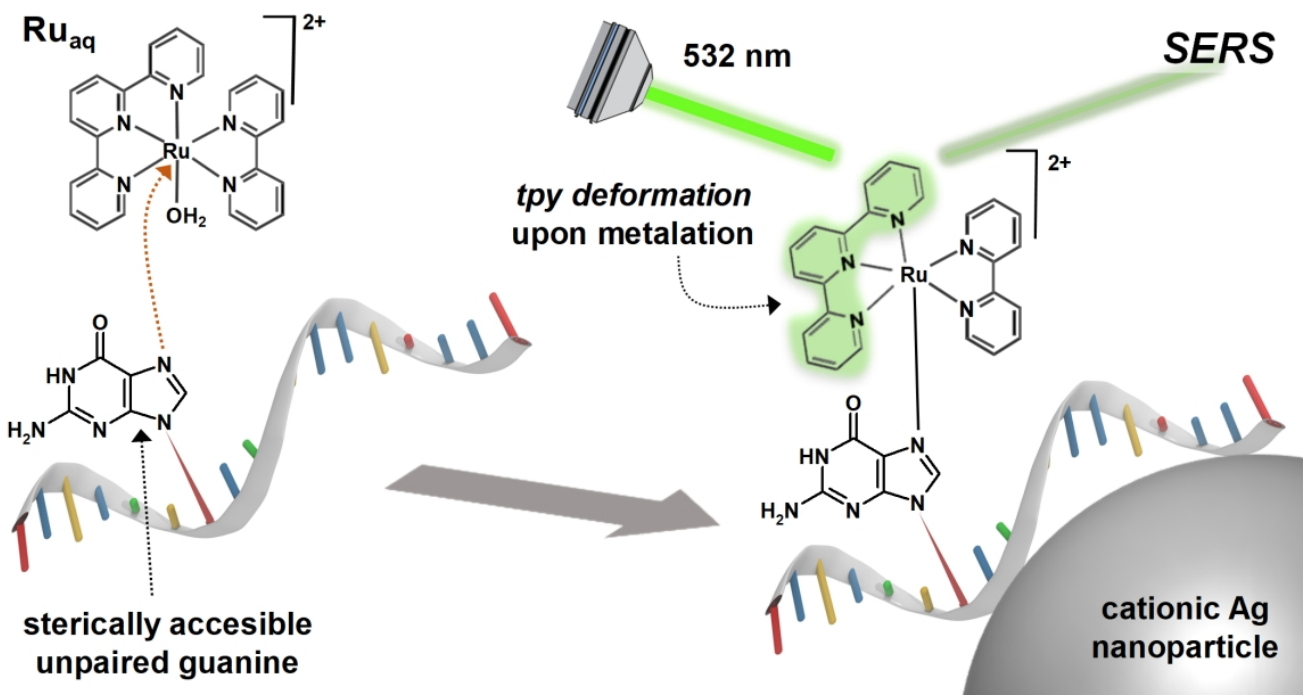

Scheme 1

$377 \times 200 \mathrm{~mm}(141 \times 141 \mathrm{DPI})$ 


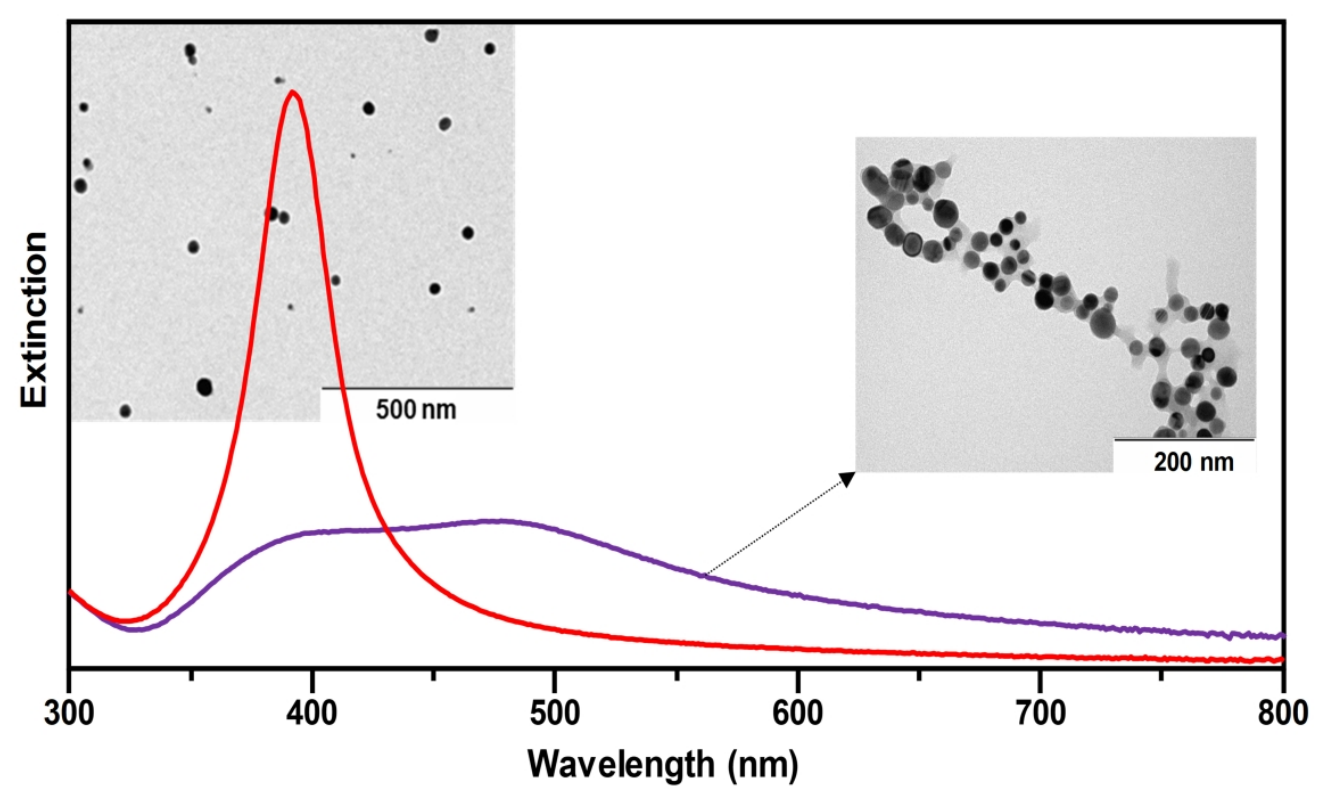

Figure 1

$1541 \times 836 \mathrm{~mm}(135 \times 150$ DPI $)$ 


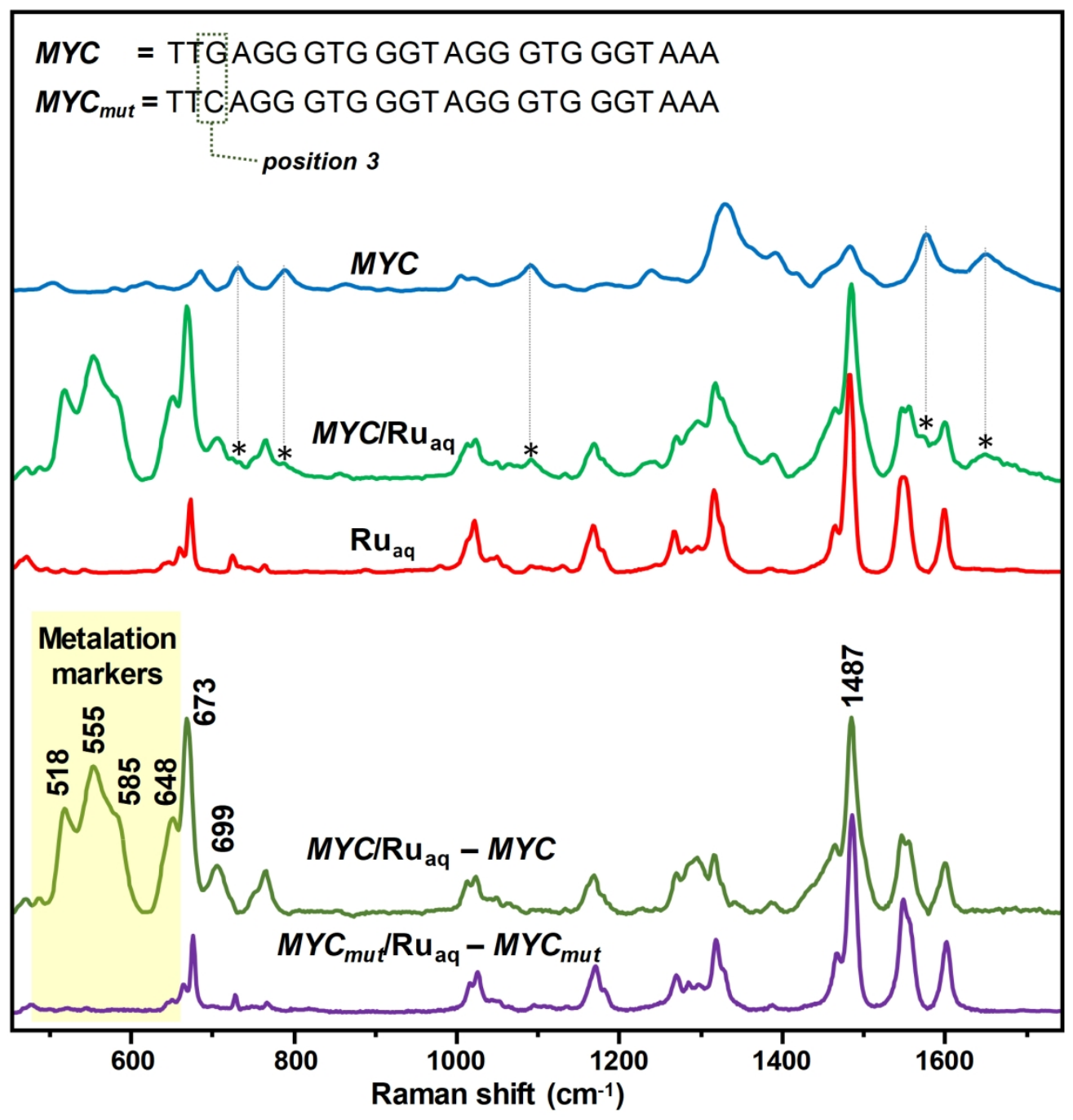

Figure 2

$364 \times 380 \mathrm{~mm}(141 \times 141 \mathrm{DPI})$ 


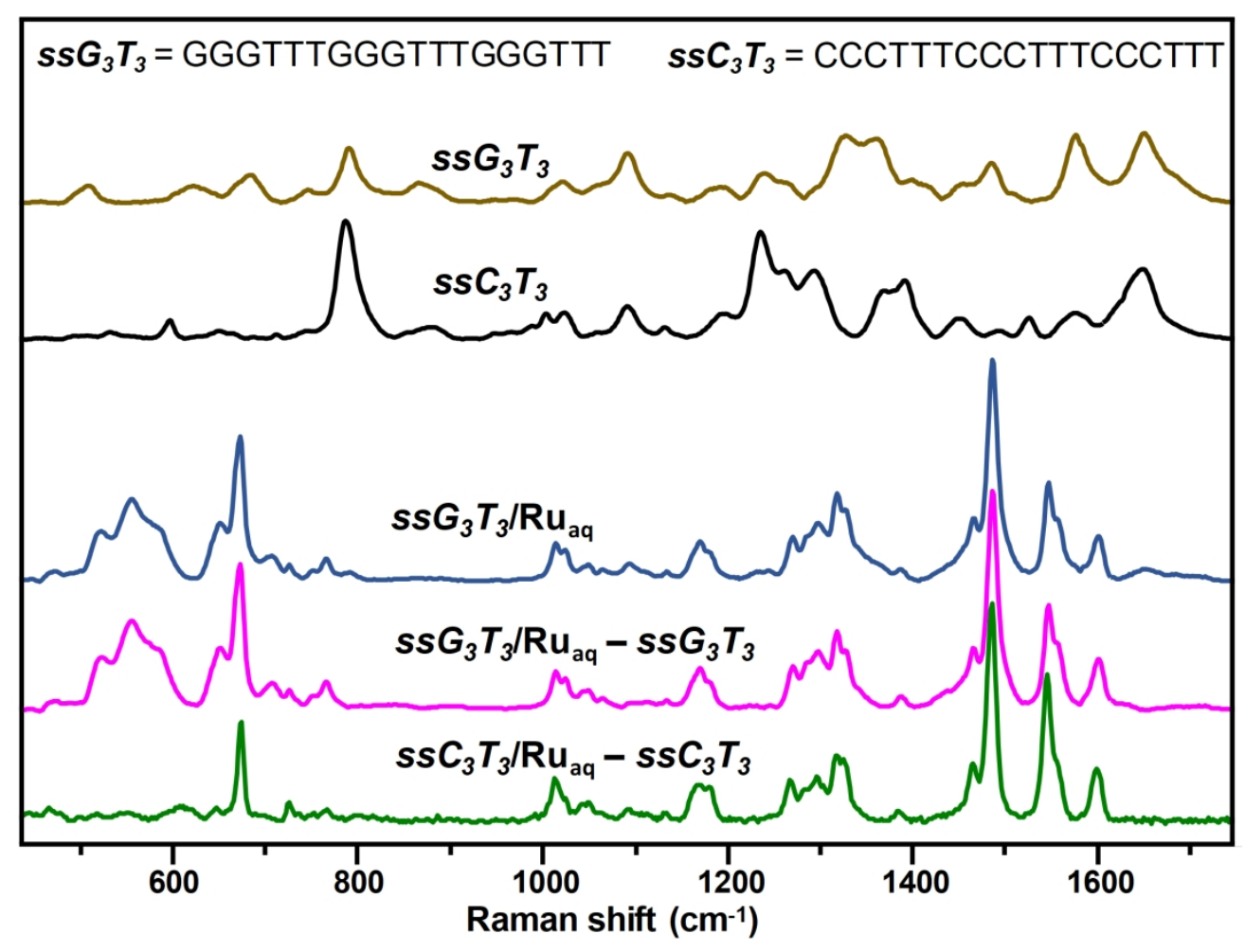

$393 \times 300 \mathrm{~mm}(141 \times 141 \mathrm{DPI})$ 


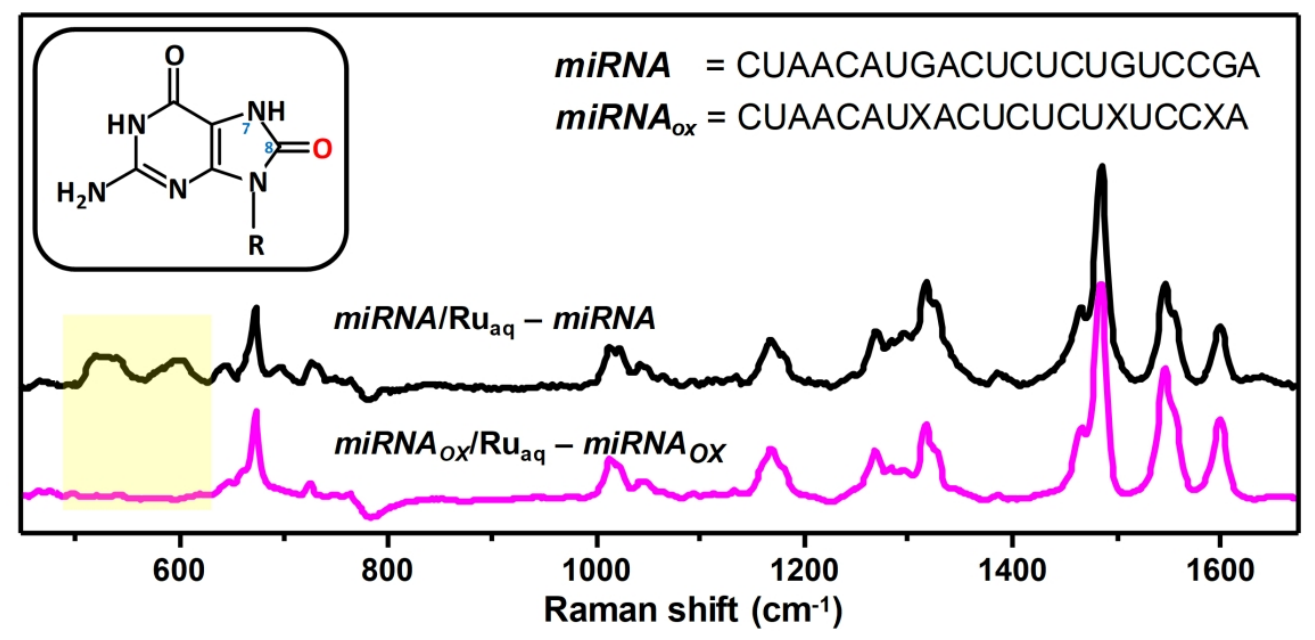

$442 \times 213 \mathrm{~mm}(141 \times 141 \mathrm{DPI})$ 